\title{
Implementation of a Spatial Channel Model for ns-3
}

\author{
Tommaso Zugno, Michele Polese \\ University of Padova \\ Padova, Italy \\ \{zugnotom,polesemi\}@dei.unipd.it
}

\author{
Natale Patriciello, Biljana Bojović, Sandra Lagen \\ Centre Tecnològic de Telecomunicacions \\ Castelldefels, Barcelona, Spain \\ \{npatriciello,bbojovic,slagen\}@cttc.es
}

\author{
Michele Zorzi \\ University of Padova \\ Padova, Italy \\ zorzi@dei.unipd.it
}

\begin{abstract}
The next generation of wireless networks will feature a more flexible radio access design, integrating multiple new technological solutions (e.g., massive Multiple-Input Multiple-Output (MIMO), millimeter waves) to satisfy different verticals and use cases. The performance evaluation of these networks will require more complex models to represent the interactions of different components of the networks accurately. For example, channel models, which are of paramount importance to precisely characterize the behavior of such systems, need to account for multi-antenna systems and new frequency bands. This paper presents the ns-3 implementation of a spatial channel model for the $0.5-100 \mathrm{GHz}$ spectrum, following the 3GPP Technical Report 38.901. The code, designed to be flexible and easily extensible, is integrated in ns-3's antenna, propagation and spectrum models, and offers the support for the investigation of future wireless systems in ns-3.
\end{abstract}

\section{CCS CONCEPTS}

\section{- Networks $\rightarrow$ Network simulations; Mobile networks. KEYWORDS}

ns-3, NR, 3GPP, mmWave, spectrum, channel model

\section{ACM Reference Format:}

Tommaso Zugno, Biljana Bojović, and Michele Zorzi. 2020. Implementation of a Spatial Channel Model for ns-3. In 2020 Workshop on ns-3 (WNS3 2020), fune 17-18, 2020, Gaithersburg, MD, USA. ACM, New York, NY, USA, 8 pages. https://doi.org/10.1145/3389400.3389401

\section{INTRODUCTION}

Wireless networks are rapidly evolving to meet the datarate, latency and reliability demands of an increasingly connected society. The 5 th generation $(5 \mathrm{G})$ of cellular networks is being deployed, following the specifications of 3rd Generation Partnership Project (3GPP) New Radio (NR) [3]. Similarly, multiple generations of Wireless Local Area Networks (WLANs) are being proposed to achieve higher capacity, improved spatial reuse, or lower energy consumption with IEEE $802.11 \mathrm{ax} / \mathrm{ay} / \mathrm{ah} / \mathrm{bd}$ [17, 42]. These wireless networking standards will be the first to exploit new technologies such as, for example, massive Multiple-Input Multiple-Output (MIMO) and millimeter wave (mmWave) communications [10].

Permission to make digital or hard copies of all or part of this work for personal or classroom use is granted without fee provided that copies are not made or distributed for profit or commercial advantage and that copies bear this notice and the full citation on the first page. Copyrights for components of this work owned by others than ACM must be honored. Abstracting with credit is permitted. To copy otherwise, or republish to post on servers or to redistribute to lists, requires prior specific permission and/or a fee. Request permissions from permissions@acm.org.

WNS3 2020, June 17-18, 2020, Gaithersburg, MD, USA

(C) 2020 Association for Computing Machinery.

ACM ISBN 978-1-4503-7537-5/20/06 ..\$15.00

https://doi.org/10.1145/3389400.3389401
Standardization bodies (i.e., the 3GPP and the IEEE) are aiming at addressing the requirements of multiple verticals and use cases with a single design of the Radio Access Network (RAN) [2]. For example, 3GPP NR can be configured to support enhanced mobile broadband (to provide ultra-high capacity to the end users), ultra-reliable and low latency communications (e.g., for remote control scenarios), and massive machine-type deployments. Therefore, future wireless networks will exhibit an increasing degree of complexity and flexibility. The different versions of IEEE WLANs and 3GPP NR can (i) operate on a wide portion of spectrum, which includes the traditional bands below $6 \mathrm{GHz}$ and the mmWave frequencies; (ii) be deployed on devices equipped with quasi-omnidirectional antennas, or with phased antenna arrays to perform analog, hybrid or digital beamforming; and (iii) support devices that communicate while moving at different speeds, up to $500 \mathrm{~km} / \mathrm{h}$ for NR.

A correct and reliable testing and performance evaluation of such complex networks becomes of paramount importance to identify the critical elements of the system before commercializing it, and to understand which algorithms and network architectures can provide the best quality of service to the end users. Simulation will play a fundamental role in this, as testbeds for $5 \mathrm{G}$ and nextgeneration WLANs are still in the making [30, 36]. Additionally, simulations can adapt better than testbeds to the large number of evolving use cases and deployment scenarios that such networks will serve. ns-3 is well positioned to be an important simulation tool for future wireless networks, thanks to the already available modules for mmWaves and NR [25, 29], IEEE 802.11ad/ay [6, 8], and to the activity to extend the wi fi module to also support IEEE 802.11ax [21].

Nonetheless, ns-3 is currently lacking common channel model Application Programming Interfaces (APIs) that can be used by all the aforementioned modules, to provide results based on the same channel abstraction, or to test the coexistence of different technologies in the same frequency spectrum. These modules, indeed, currently use different channel modeling techniques, included in the modules themselves [7, 34, 41], not directly comparable with each other, and not designed with a modular and extensible approach. ns-3, on the other hand, provides a number of propagation models, and a flexible abstraction for the spectrum usage of single and multi carrier systems [9], but is lacking a fading model that can be integrated with multi-antenna wireless technologies.

The channel model, however, is one of the most important components of a wireless network simulator, as the results can only be as accurate as the channel model [14]. In particular, when it comes to mmWaves, the harsh propagation conditions may severely impact the performance of the higher layers of the protocol stack, much more so than at traditional sub-6 GHz frequencies [31]. Moreover, mmWave systems generally exploit beamforming to increase 
the link budget of the communication, and this element has to be introduced in the overall modeling process of the channel. Additionally, when considering MIMO systems, an exact characterization of the rank of the wireless channel is necessary for a proper evaluation of how many parallel streams can be supported [38].

In this paper, we present the implementation of a channel model for future wireless networks that has been recently included in ns-3. Notably, we implemented a Spatial Channel Model (SCM) for the spectrum module, which characterizes the channel through a matrix $\mathbf{H}$, in which each single entry models the channel between two antenna elements at the transmitter and the receiver [37]. The channel realization is computed using the 3GPP stochastic model for $5 \mathrm{G}$ networks between 0.5 and $100 \mathrm{GHz}$ [4]. Additionally, we extended the propagation module to support the models in [4], with a different characterization for Line of Sight (LOS) and Non Line of Sight (NLOS) states (according to whether the direct path between the transmitter and the receiver is blocked or not), and the antenna module, which now features antenna arrays. The implementation of the channel model equations is based on that in [41], but the code has been refactored and redesigned to be as modular as possible, with a clear separation of the propagation model, the fading, the antenna, and the beamforming. Moreover, it can be easily extended to support other fading models based on the computation of a channel matrix. We believe that this model, which has been developed as part of the Google Summer of Code project, represents a substantial and timely contribution to the wireless research community that uses ns-3 to study next-generation wireless networks.

The rest of the paper is organized as follows. In Section 2 we review modern channel modeling efforts, with a focus on the 3GPP channel model for $5 \mathrm{G}$ and on why SCMs are widely used in this context. We describe the implementation of the 3GPP SCM from [4] in Section 3, and present examples and comment on use cases in Section 4. Finally, we conclude the paper in Section 5.

\section{RECENT DEVELOPMENTS IN CHANNEL MODELING}

Channel modeling is a fundamental activity for the design and evaluation of future wireless networks. The authors of [14] claim that the new features of cellular and WLAN networks call for new approaches in channel modeling. Large antenna arrays and the deployment of MIMO techniques require the addition of the spatial dimension in the channel, with a full 3D model, capable of characterizing the diversity of the channel paths for each pair of antenna elements between the transmitter and the receiver. Moreover, the channel in the new frequency bands of 3GPP NR and IEEE 802.11ad/ay (i.e., mmWaves) needs proper understanding, especially with respect to multipath fading and blockage. Finally, new deployments (e.g., vehicular networks) introduce additional modeling requirements for network simulations.

These challenges have motivated several efforts in channel modeling, especially when considering mmWave frequencies [16]. Multiple measurement campaigns in these frequency bands have strived to accurately model the propagation and fading in different scenarios [18, 32, 35], highlighting how mmWaves are characterized by high propagation loss, sensitivity to blockage, and a reduced impact of small scale fading with sparsity in the angular domain. These measurement campaigns have then led to different families of channel models for future wireless networks, generally given by the combination of propagation loss and fading models. The different modeling approaches differ for their degree of abstraction, simplicity and accuracy. Analytical studies for $5 \mathrm{G}$ generally use simple propagation loss models, combined with Nakagami-m or Rayleigh fading [5]. These models are computationally efficient, but fail to capture the spatial dimension of the channel and cannot be combined with realistic beamforming models. Quasi-deterministic channels, developed, for example, for IEEE 802.11ad/ay [24], are instead designed to be as accurate as possible in specific scenarios, but are much more complex and require a precise characterization of the environment [22].

\subsection{GPP TR 38.901}

For the evaluation of NR, the 3GPP has adopted a 3D SCM [4], which represents a tradeoff between the two aforementioned channel modeling approaches: it is generic, thanks to its stochastic nature, but at the same time can model interactions with beamforming vectors. An SCM, indeed, models the channel through a channel matrix $\mathbf{H}(t, \tau)$, with as many rows and columns as the number of transmit $(U)$ and receive $(S)$ antenna elements. Each entry $H_{u, s}(t, \tau)$ corresponds to the impulse response of the channel between the $s$-th element of the Base Station (BS) antenna and the $u$-th element of the User Terminal (UT) antenna at delay $\tau$ at time $t . H_{u, s}(t, \tau)$ is generated by the superposition of $N$ different clusters, representing groups of multipath components that arrive and/or depart the antenna arrays with certain angles. The multipath components impact the receiving array with different delays, and the power will be scaled according to a delay-based profile. If present, an LOS cluster is modeled with the strongest power and the minimum delay. The other clusters, instead, represent reflections from the scattering environment.

The 3GPP channel modeling framework is described in TR 38.901 [4] and represents the extension of TR 38.900, which was targeted for above- $6 \mathrm{GHz}$ bands only. It supports the modeling of wireless channels between 0.5 and $100 \mathrm{GHz}$ by means of a stochastic SCM, in which a single instance of the channel matrix $\mathbf{H}(t, \tau)$ is computed according to random distributions for large scale fading parameters (i.e., the delay profile, the angles of arrival and departure, and the shadowing) and for the small scale fading (i.e., for small variations in the channel, for example, as given by the Doppler spread). To enable the simulation of signal propagation in different environments, it specifies four scenarios, with different parameters for the random distributions underlying the channel:

- RMa (Rural Macro), targeting rural deployments with continuous wide area coverage;

- UMa (Urban Macro), intended to model urban areas with macrocells mounted above the rooftops of the surrounding buildings;

- UMi (Urban Micro) Street Canyon, similar to UMa but with base stations mounted below the rooftops;

- Indoor Hotspot (InH) Mixed and Open Office, to model indoor environments.

For each scenario, this model provides the characterization of the LOS/NLOS channel condition, the propagation loss, and the small scale fading due to the effect of Doppler and multipath. Also, it 


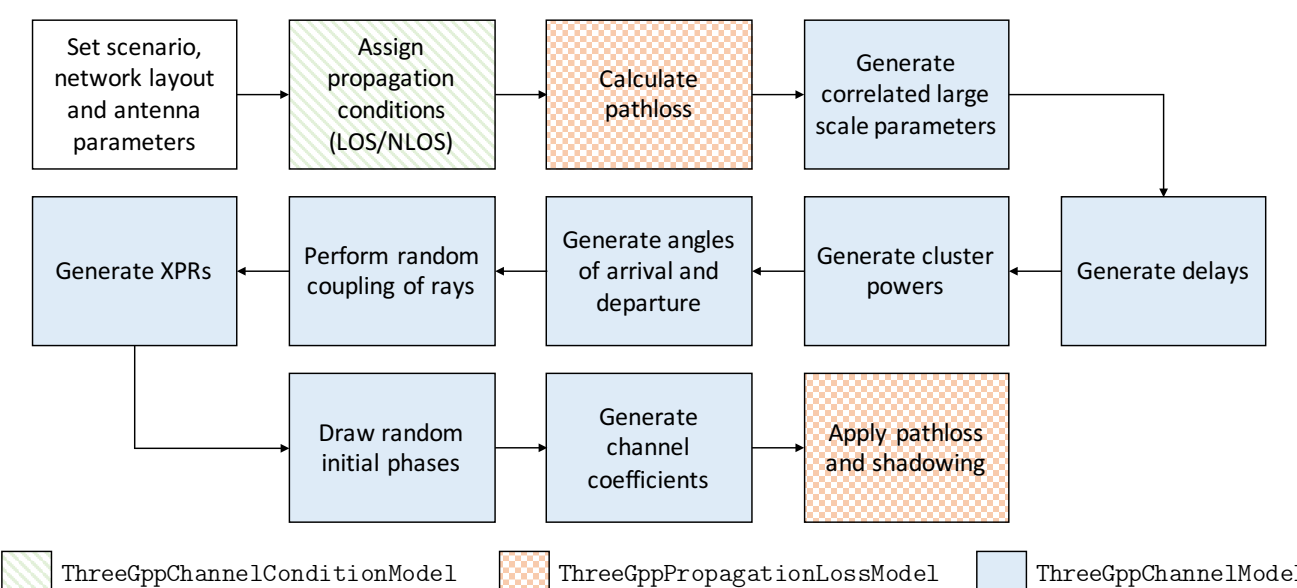

Figure 1: Diagram Representing the Channel Generation Procedure; the Colors Indicate the Classes where the Steps are Accomplished

defines a radiation model to account for the non-isotropic behavior of real antennas.

The channel matrix generation procedure, represented in Figure 1, accounts for both large (i.e., pathloss and shadowing) and small scale (fast fading) propagation phenomena, and provides the possibility to select different models and parameters depending on the scenario of interest. The pathloss model describes the signal attenuation between the transmitter and the receiver as a function of the 3D positions and the carrier frequency. The shadowing model provides the statistical characterization of the attenuation due to the presence of obstacles between the transmitter and the receiver. The small scale fading accounts for the signal phase and amplitude variations due to small changes in the spatial separation between the transmitter and the receiver, and for the Doppler effect introduced by a moving terminal. While the large scale propagation effects are considered to be constant within the frequency band of interest, the small scale fading has a frequency-selective behavior, thus introducing a gain which varies within the band.

In the following, we describe the 3GPP SCM for $5 \mathrm{G}$ networks that has been implemented in ns-3, providing details on the pathloss and channel condition computations, the channel matrix generation procedure, and the antenna model that can be associated to such matrix.

\section{NS-3 IMPLEMENTATION}

In ns-3, the modeling of the signal propagation through the wireless channel is handled by the spectrum module, which includes the abstract classes SpectrumPhy and SpectrumChannel. Devices communicating through the same wireless channel have their own SpectrumPhy instances, which are in charge of creating the Power Spectral Density (PSD) of the transmitted signals. The different SpectrumPhy instances are attached to the same SpectrumChannel object which dispatches the transmissions among the devices. At each transmission, SpectrumPhy calls the method SpectrumChannel: : StartTx which notifies each receiver and computes the corresponding PSDs of the received signals. To account for the power attenuation and fading due to the propagation of the signal through the environment, SpectrumChannel relies on two standard interfaces, i.e., PropagationLossModel and SpectrumPropagationLossModel. The former models slow fading, in which the loss is constant over the frequency band of the signal, while the latter is used for fast fading models, which introduce frequencyselective losses.

The 3GPP SCM can be divided into four main components, namely, (i) channel condition models, used to determine the LOS/NLOS channel state, (ii) propagation loss models, including pathloss and shadowing, (iii) the fast fading model, and (iv) the antenna model. The objective of this project was to implement these components using, whenever possible, the interfaces provided by the spectrum and propagation modules [9], without compromising the support of existing models and ensuring an easy integration in the main code base. We decided to implement each component as a separate class in order to achieve a flexible and re-usable architecture, enabling the possibility to easily replace, modify or include new parts. Figure 2 reports a simplified UML diagram for the classes involved in the channel model implementation.

\subsection{LOS Probability Models}

The first step for the generation of the channel matrix is to determine the LOS/NLOS channel condition. 3GPP TR 38.901 provides stochastic models to determine the channel state in all the scenarios of interest, taking into account the distance between the communication endpoints and the characteristics of the propagation environment, e.g., the presence of buildings and obstacles.

Since ns-3 lacks a general way to account for the channel state, we developed the class ChannelCondition, which stores the state information related to a certain channel. Also, we proposed a new interface, called ChannelConditionModel, which can be extended to implement any specific channel condition model, either stochastic or deterministic. The main method is GetChannelCondition, which accepts as argument the positions of the two nodes and returns a pointer to the corresponding ChannelCondition instance.

To include the channel condition models defined in the 3GPP TR 38.901, we developed five different classes, i.e., ThreeGppRmaChannelConditionModel, ThreeGppUmaChannelConditionModel, ThreeGppUmiStreetCanyonChannelConditionModel, ThreeGppIndoorOpenOfficeChannelConditionModel and ThreeGppIndoorMixedOfficeChannelConditionModel, each handling a different scenario. All the new classes derive from the same base, called ThreeGppChannelConditionModel, which extends the ChannelConditionModel interface and provides caching functionalities for the periodic update of the states. When the method GetChannelCondition is called for the first time, the channel state is computed 


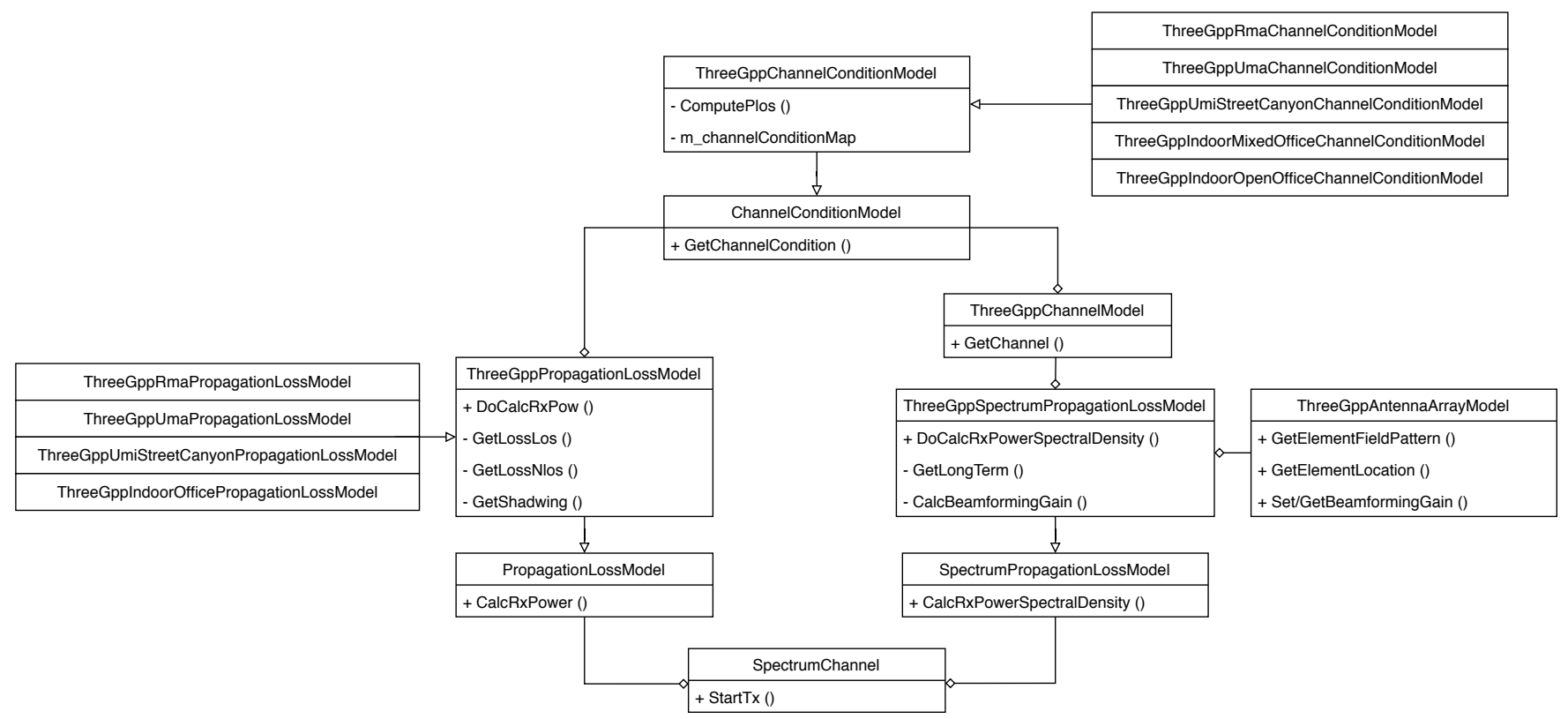

Figure 2: Simplified Unified Modeling Language (UML) Diagram of the SCM Implementation

and its value is stored in a map, together with the generation time. Then, at subsequent calls, the method checks if the state has to be updated or not based on the time expired since its generation and, if so, a new state is independently generated, without accounting for any temporal correlation. The update interval can be tuned by the user with the attribute UpdatePeriod, with the possibility of never updating the channel condition if the attribute is set to 0 .

\subsection{Pathloss and Shadowing Models}

The pathloss models defined in 3GPP TR 38.901 can be expressed through the general form of Eq. (1), where $d$ is the 3D distance between the two endpoints, $f_{C}$ is the carrier frequency, $A, B$ and $C$ are model parameters, and $X$ is an optional loss term.

$$
P L=A \log _{10}(d)+B+C \log _{10}\left(f_{C}\right)+X \quad[\mathrm{~dB}]
$$

In particular, $A$ represents the pathloss exponent and accounts for the dependence on the distance between the receiver and the transmitter, while $C$ determines the relation between the pathloss and the carrier frequency. $A, B, C$ and $X$ take different values depending on the propagation conditions, such as the scenario, the LOS/NLOS channel state and the break point distance $d_{B P}$, as defined in [4].

Also, to account for the variations of the received signal power due to blockage events, a log-normal shadowing component is added to the mean pathloss. Adjacent fading values are correlated with an exponential autocorrelation function, and their correlation depends on the spatial separation between the two positions. As for the pathloss, the standard deviation of the shadowing component, as well as the autocorrelation function, depend on the specific propagation conditions.

Moreover, 3GPP TR 38.901 specifies a model to account for the outdoor-to-indoor penetration loss due to buildings or cars, which however was not considered in this work and is planned for future development.
To include the pathloss and shadowing model defined in 3GPP TR 38.901, we developed the base class ThreeGppPropagationLossModel, which extends the PropagationLossModel interface and implements the general logic used to handle the computation of the mean pathloss and the shadowing component. Then, we extended this class by developing four subclasses, i.e., ThreeGppRmaPropagationLossModel, ThreeGppUmaPropagationLossModel, ThreeGppUmaStreetCanyonPropagationLossModel and ThreeGppIndoorOfficePropagationLossModel, which define the models for the different channel scenarios. Since the propagation loss depends on the LOS/NLOS channel state, the ThreeGppPropagationLossModel class is paired with a channel condition model through the ChannelConditionModel interface. The main method is DoCalcRxPower, which returns the power received at the receiver side based on the positions of the communicating nodes. It makes use of the methods GetLossLos and GetLossNlos to compute the mean pathloss in the LOS and NLOS states, respectively, and of the method GetShadowing to apply the shadowing model. Two other functions, namely GetShadowingStd and GetShadowingCorrelationDistance, are used by GetShadowing to retrieve the standard deviation of the shadowing component and the correlation distance, a parameter which defines the autocorrelation function.

\subsection{Fast Fading Model}

The fast fading model included in 3GPP TR 38.901 accounts for the changes in the phase and amplitude of the transmitted signal due to the effect of multipath propagation, i.e., the presence of multiple signal components that propagate over different paths. It provides the possibility to set the model parameters depending on the scenario of interest, thus enabling the modeling of multiple propagation environments.

Eq. (2) represents the overall channel impulse response $H_{u, s}(t, \tau)$. As mentioned in Section 2, it is obtained by the superposition of $M \times N$ rays, grouped in $N$ clusters. Rays belonging to the same cluster experience the same power $P_{n}$ and propagation delay $\tau_{n}$, present 
Table 1: Main Entries of ThreeGppChannelMatrix

\begin{tabular}{|ll|}
\hline & ThreeGppChannelMatrix \\
\hline m_channel & the channel coefficients $H_{u, s, n}$ \\
m_delay & the clusters delays $\tau_{n}$ \\
m_angle & the clusters arrival and departure angles \\
m_generatedTime & a time stamp indicating the generation time \\
m_nodeIds & IDs of the transmitter and receiver nodes \\
\hline
\end{tabular}

similar angles of arrival $\left(\theta_{n, m}^{A}, \phi_{n, m}^{A}\right)$ and departure $\left(\theta_{n, m}^{D}, \phi_{n, m}^{D}\right)$, and have uniformly distributed initial phases $\Phi_{n, m}$. Each ray accounts for the antenna field patterns $\mathbf{F}\left(\theta_{n, m}, \phi_{n, m}\right)$ and for the power distribution among the vertical and horizontal polarizations through the term $K_{n, m}$. The terms $\exp \left(j \overline{\mathbf{k}}^{T} \overline{\mathbf{d}}\right)$ represent the array responses of the transmitting and receiving antennas, where $\overline{\mathbf{k}}$ is the wave vector and $\overline{\mathbf{d}}$ is the element location vector. In case of user mobility, each ray is subject to a phase shift $v_{n, m}$ due to the Doppler effect. In the LOS case, a Ricean factor is added to the direct path.

$$
\begin{aligned}
& H_{u, s}(t, \tau)=\sum_{n=1}^{N} \sqrt{\frac{P_{n}}{M}} \sum_{m=1}^{M} \overline{\mathbf{F}}_{r x}\left(\theta_{n, m}^{A}, \phi_{n, m}^{A}\right) \\
& \times\left[\begin{array}{cc}
e^{j \Phi_{n, m}^{\theta, \theta}} & \sqrt{K_{n, m}^{-1}} e^{j \Phi_{n, m}^{\theta, \phi}} \\
\sqrt{K_{n, m}^{-1}} e^{j \Phi_{n, m}^{\phi, \theta}} & e^{j \Phi_{n, m}^{\phi, \phi}}
\end{array}\right] \\
& \times \overline{\mathbf{F}}_{t x}\left(\theta_{n, m}^{D}, \phi_{n, m}^{D}\right) \\
& \times e^{j \overline{\mathbf{k}}_{r x, n, m}^{T} \overline{\mathbf{d}}_{r x, u}} e^{j \overline{\mathbf{k}}_{t x, n, m}^{T} \overline{\mathbf{d}}_{t x, s}} \\
& \times e^{j 2 \pi v_{n, m} t} \delta\left(\tau-\tau_{n}\right)
\end{aligned}
$$

Our implementation follows the same approach described in [41], but introduces some changes to improve the modularity of the code and includes the latest updates with respect to [1]. As in [41], to reduce the model complexity, we assumed that all rays within a cluster are subject to the same Doppler shift $\left(v_{n}\right)$, corresponding to that of the central ray. Thus, the channel impulse response can be expressed as:

$$
H_{u, s}(t, \tau)=\sum_{n=1}^{N} H_{u, s, n} e^{j 2 \pi v_{n} t} \delta\left(\tau-\tau_{n}\right),
$$

where $H_{u, s, n}$ represents all the terms of the impulse response except for the Doppler contribution.

We developed the class ThreeGppChannelModel, which computes the coefficients $H_{u, s, n}$ as described in Section 7.5 of [4] and handles their periodic update. It is associated with an instance of ChannelConditionModel, used to determine the LOS/NLOS channel state. The main method is GetChannel, which takes as input the mobility models of the transmitter and receiver nodes and the associated antenna objects, and returns an instance of ThreeGppChannelMatrix. As represented in Table 1, the structure ThreeGppChannelMatrix contains entries to store the channel coefficients $H_{u, s, n}$, the propagation delays $\tau_{n}$, the angles of arrival and departure, and a time stamp indicating the generation time. The first time a channel is generated, the corresponding ThreeGppChannelMatrix is cached in a map together with identifiers for the transmitting and receiving nodes. When the same channel is requested again, the method GetChannel retrieves the ThreeGppChannelMatrix from the map and checks whether the channel coefficients have to be updated or not, depending on the expired time and the occurrence of LOS-NLOS transitions. If so, it recomputes the coefficients, otherwise it returns the old realization. Moreover, the class ThreeGppChannelModel provides attributes to enable an easy configuration of the model parameters, such as carrier frequency, channel scenario and update period. In particular, the choice of the update period should consider (i) the channel coherence time, i.e., the time duration over which the channel response does not vary, which depends on several factors, such as frequency, user mobility and propagation environment, and (ii) the time granularity of the simulation, which should be fine enough to capture the channel dynamics.

3.3.1 Blockage Model. 3GPP TR 38.901 also provides an optional feature that can be used to model the blockage effect due to the presence of obstacles, such as trees, cars or humans, at the level of a single cluster. This differs from a complete blockage, which would result in an LOS to NLOS transition. Therefore, when this feature is enabled, an additional attenuation is added to certain clusters, depending on their angle of arrival. There are two possible methods for the computation of the additional attenuation, i.e., stochastic (Model A) and geometric (Model B). In this work, we used the implementation provided by Zhang et al. in [41], which uses the stochastic method. In particular, we extended the class ThreeGppChannelModel by including the method CalcAttenuationOfBlockage, which computes the additional attenuation. Also, we defined attributes to enable/disable the blockage feature and to configure the model parameters.

\subsection{Antenna Array Model}

In 3GPP TR 38.901, BS and UT antennas are modeled as uniform rectangular antenna arrays with multiple panels, each containing $N_{a, c} \times N_{a, r}$ antenna elements with fixed spacing, where $N_{a, c}$ is the number of elements in a column and $N_{a, r}$ is the number of elements in a row of the antenna array. Orientation of the arrays can be configured by adjusting bearing, tilt and slant angles.

The technical report [4] describes how to compute the azimuth and zenith components of the field pattern $\mathrm{F}$ to be used for the generation of the channel matrix, i.e., $F_{\theta}$ and $F_{\phi}$, respectively, taking into account orientation and polarization of the panels. We developed a new class, called ThreeGppAntennaArrayModel, which implements this model under the following assumptions: (i) the array is composed of a single panel; (ii) the slant angle is fixed and equal to 0 degrees; and (iii) the antenna elements are vertically polarized.

The main method is GetElementFieldPattern, which accepts as argument the azimuth and zenith angles of arrival and returns std: : pair containing the element field components computed following a four-step procedure. The first step is to express the azimuth and zenith angles of arrival using the antenna local coordinate system, i.e., with the origin corresponding to the bottom-left corner of the array and $\mathrm{x}$ - and $\mathrm{y}$-axes parallel to the horizontal and vertical sides, using the procedure described in Section 7.1 of [4]. The second step is to compute the radiation power pattern of each element, which describes how the irradiated power varies in space. The technical report specifies the radiation power pattern as a function of the zenith and azimuth angles of arrival $\left(\theta^{\prime}, \phi^{\prime}\right)$, 
expressed in the local coordinate system:

$$
\begin{aligned}
A_{d B}^{\prime}\left(\theta^{\prime}, \phi^{\prime}\right)= & G_{E}-\min \left\{\min \left[12\left(\frac{\theta^{\prime}-90^{\circ}}{\theta_{3 d B}}\right)^{2}, S L A_{V}\right]\right. \\
& \left.-\min \left[12\left(\frac{\phi^{\prime}}{\phi_{3 d B}}\right)^{2}, A_{\max }\right], A_{\max }\right\},
\end{aligned}
$$

where $\theta_{3 d B}$ and $\phi_{3 d B}$ represent the vertical and horizontal beamwidth and are equal to $65^{\circ}, S L A_{V}$ is the side-lobe level limit and is set to $30 \mathrm{~dB}, A_{\max }$ represents the front-back ratio and is fixed to $30 \mathrm{~dB}$, while $G_{E}$ represents the directional gain of an antenna element and is a model parameter. The third step is to derive the azimuth and zenith components of the element field pattern. Since only vertical polarization is considered, we have $F_{\phi^{\prime}}^{\prime}\left(\theta^{\prime}, \phi^{\prime}\right)=0$ and $F_{\theta^{\prime}}^{\prime}\left(\theta^{\prime}, \phi^{\prime}\right)=\sqrt{A^{\prime}\left(\theta^{\prime}, \phi^{\prime}\right)}$. Finally, the fourth step is to convert the field pattern components back to the global coordinate system to obtain $F_{\theta}(\theta, \phi)$ and $F_{\phi}(\theta, \phi)$.

The ThreeGppAntennaArrayModel class features also the method GetElementLocation, which accepts as argument the index of the antenna element and returns the corresponding location vector $\overline{\mathbf{d}}$, and provides the methods Set/GetBeamformingVector to store and retrieve the beamforming vector $\mathbf{w}$.

\subsection{Computation of the PSD}

The PSD of the received signal is computed as:

$$
S_{r x}(t, f)=S_{t x}(t, f) \mathbf{w}_{r x}^{T} \mathcal{H}(t, f) \mathbf{w}_{t x},
$$

where $S_{t x}(t, f)$ is the PSD of the transmitted signal, $\mathbf{w}_{r x}$ and $\mathbf{w}_{t x}$ are the transmitting and receiving beamforming vectors, and $\mathcal{H}(t, f)$ is the channel matrix in the frequency domain. Applying the Fourier transform to channel coefficients expressed as in Eq. (3), $S_{r x}(t, f)$ can be rewritten as:

$$
\begin{aligned}
S_{r x}(t, f) & =S_{t x}(t, f) \sum_{n=1}^{N} \sum_{s=1}^{S} \sum_{u=1}^{U} w_{r x, u} H_{u, s, n} w_{t x, s} e^{j 2 \pi v_{n} t} e^{j 2 \pi \tau_{n} f} \\
& =S_{t x}(t, f) \sum_{n=1}^{N} L_{n} e^{j 2 \pi v_{n} t} e^{j 2 \pi \tau_{n} f},
\end{aligned}
$$

where $L_{n}$ represents the long-term component of cluster $n$, as defined in [41].

In our implementation, the computation of $S_{r x}(t, f)$ is handled by the class ThreeGppSpectrumPropagationLossModel, which extends the SpectrumPropagationLossModel interface. This class interacts with ThreeGppChannelModel to retrieve the channel coefficients and holds a map containing the ThreeGppAntennaArray objects of all the devices. The main method is DoCalcRxPowerSpectralDensity, which takes as input the mobility models of transmitter and receiver nodes, and returns the PSD of the received signal, computed using Eq. (6). In particular, it relies on the private methods GetLongTerm, to calculate the long term components, and CalcBeamformingGain, to account for the Doppler and the propagation delay. To reduce the computational load, all the long term components associated with a certain channel are cached and recomputed only when the channel realization is updated. Also, ThreeGppSpectrumPropagationLossModel provides the method
SetChannelModelAttribute, which can be used to configure the model parameters, such as carrier frequency and channel scenario.

\section{EXAMPLES AND USE CASES}

In this section, we describe, by means of an example, how the different classes presented above have to be configured to build the entire 3GPP channel modeling framework. Also, we outline some possible use cases.

\subsection{Examples}

The example three-gpp-channel-example.cc included in the spectrum module demonstrates the usage of the proposed framework. It involves two devices, a transmitter and a receiver, placed at a certain distance from each other and communicating over a wireless channel. At regular intervals, we simulate a transmission between the two nodes and estimate the Signal to Noise Ratio (SNR) perceived at the receiver node. The script provides the possibility to configure the distance between the two nodes, the channel model parameters, as well as the transmission power and the receiver noise figure. Also, it produces an output trace containing the experienced propagation loss and the SNR estimate. As an example, in Figure 3 we reported the average propagation loss (solid lines) over the distance between the two devices operating at $2.1 \mathrm{GHz}$ for different scenarios and channel conditions, obtained by averaging the results of 100 independent runs of this script. The black dashed lines represent the pathloss value computed from the models defined in 3GPP TR 38.901.

In the following, we review the procedure used to create and configure the channel model classes, assuming that the UMa scenario is selected:

(1) create an instance of the class ThreeGppUmaChannelConditionModel;

(2) create an instance of ThreeGppUmaPropagationLossModel, configure the carrier frequency through the attribute "Frequency", and set the channel condition model through the attribute "ChannelConditionModel";

(3) create an instance of ThreeGppSpectrumPropagationLossModel, set the UMa scenario, the carrier frequency and the channel condition model through the method SetChannelModelAttribute and using the attributes "Scenario", "Frequency" and "ChannelConditionModel";

(4) for each device, create an instance of ThreeGppAntennaArrayModel and inform the ThreeGppSpectrumPropagationLossModel class about the device-antenna associations by calling the method ThreeGppspectrumPropagationLossModel : : AddDevice.

Besides being implemented in the three-gpp-channel-example.cc script, these steps could be included in a helper class for an ns-3 module that aims at using this channel model. For a proper usage of this model, users may need to set the transmission time granularity of the simulation based on the channel coherence time of the scenario of interest and use an error model that accounts for the non-Additive White Gaussian Noise (AGWN) behavior of fast fading channels. For example, the error model in [20], which has been developed according to TR 38.901, could be used in combination with the proposed channel model for NR system-level 


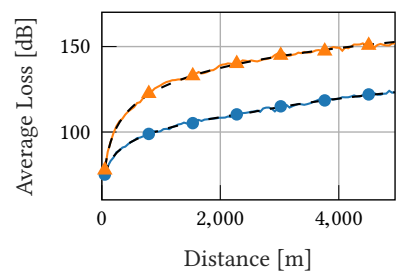

(a) RMa

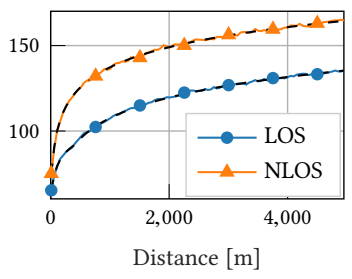

(b) UMa

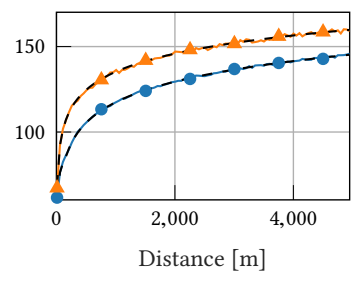

(c) UMi Street Canyon

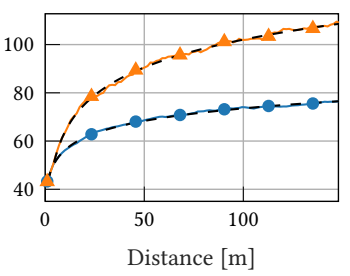

(d) Indoor Office (Mixed)

Figure 3: Average Propagation Loss vs Distance between the Nodes

simulations, provided that the channel coherence time is larger than the slot length of the NR frame structure.

\subsection{Use Cases}

The main target of the developed model is to enable system-level simulations of $3 \mathrm{GPP}$ scenarios through a $3 \mathrm{GPP}$-compliant channel and antenna model. As such, it is a requirement for any 3GPP LTE and NR-based system-level simulation that aims to properly model and evaluate the performance of physical layer techniques using appropriate channel modeling, both in the sub- $6 \mathrm{GHz}$ bands and in mmWave bands.

Moreover, it enables the simulation and coexistence studies of different technologies that share the spectrum resources, such as 3GPP and IEEE Radio Access Technologies (RATs) in unlicensed/dedicated spectrum bands. For example, it can be used to evaluate the 3GPP and IEEE RATs coexistence of:

- IEEE $802.11 \mathrm{~b} / \mathrm{g} / \mathrm{a} / \mathrm{n} / \mathrm{ac} / \mathrm{ax}$ (Wi-Fi) and 3GPP LTE-LAA (Licensed-Assisted Access) in unlicensed sub-6 GHz bands [12];

- IEEE $802.11 \mathrm{~b} / \mathrm{g} / \mathrm{a} / \mathrm{n} / \mathrm{ac} / \mathrm{ax}$ (Wi-Fi) and 3GPP NR-U in unlicensed sub- $6 \mathrm{GHz}$ bands $[13,23]$;

- IEEE 802.11ad/ay (WiGig, directional multi-Gigabit Wi-Fi) and 3GPP NR-U in unlicensed $60 \mathrm{GHz}$ bands [19, 26];

- IEEE $802.11 \mathrm{p} / \mathrm{bd}$, 3GPP C-V2X (Cellular V2X) and 3GPP NR V2X (Vehicle-to-Everything) in dedicated sub-6 GHz bands [15];

- IEEE 802.11bd and 3GPP NR V2X in dedicated mmWave bands [27];

- Wi-Fi, IEEE 802.11p/bd, 3GPP C-V2X and 3GPP NR V2X in unlicensed sub-6 GHz bands [15];

- WiGig, IEEE 802.11bd and 3GPP NR V2X in unlicensed 60 $\mathrm{GHz}$ bands [27].

Also, the proposed model provides a common framework for simulations of spectrum sharing solutions through either spectrum refarming or dynamic spectrum sharing, for example, if different 3GPP RATs of the same operator share the licensed spectrum for some long period of time until one of the RATs becomes obsolete (spectrum refarming). This happens in low frequency bands (e.g., $900,1800 \mathrm{MHz}$ ) that are essential for 3GPP NR to achieve coverage, but in which 3GPP LTE is already deployed and operational, and cannot thus be migrated to other frequency bands. As such, a key example of spectrum refarming is that of 3GPP LTE and 3GPP NR in licensed sub- $6 \mathrm{GHz}$ bands. Another example of spectrum sharing is when different operators share the spectrum by means of coordination policies. In this regard, the research community has also recently proposed solutions based on spectrum sharing [33] and spectrum pooling [11] for mmWave bands, which exploit coordination among different cellular network operators to improve the spatial reuse, and which could be tested from an end-to-end perspective on top of the proposed framework.

In addition, the developed model is also useful to evaluate the 3GPP and IEEE interworking through a common channel modeling. 3GPP and IEEE interworking considers core network and radio access network integration by means of aggregating 3GPP-based RATs in licensed bands and Wi-Fi in unlicensed bands. Examples for which the developed model could be used include:

- Wi-Fi and 3GPP LTE interworking, e.g., through LTE-WLAN Aggregation (LWA) and LTE-WLAN Radio Level Integration with IPsec Tunnel (LWIP) [28],

- IEEE 802.11ax and 3GPP NR interworking [40].

\section{CONCLUSIONS AND FUTURE WORK}

In this paper, we introduced a new channel model for ns-3, developed following the specifications in [4]. This work is expected to enrich ns-3 by enabling a more accurate modeling of the dynamics of wireless channels between 0.5 and $100 \mathrm{GHz}$, thus enhancing the support for the simulation of wireless systems. In Section 1, we introduced the importance of channel models and simulation tools for the design of next-generation wireless systems. In Section 2, we explained the motivations that drive the design of more accurate channel models and described the SCMs approach. In Section 3, we overviewed the 3GPP SCM and explained how it has been implemented in ns-3. Finally, in Section 4, we provided an example of usage and outlined some possible use cases.

We plan to further improve this work by (i) refining the antenna model to enable the modeling of multiple panels with dual polarization; (ii) implementing the outdoor-to-indoor penetration loss model described in [4], Section 7.4.3; (iii) implementing the additional modeling components, such as the spatial consistency procedure and the modeling of the oxygen absorption, specified in [4], Section 7.6; (iv) performing a calibration campaign to validate the model following the assumptions reported in [4], Section 7.8; and (v) lowering the computation time needed to generate a channel realization [39] through the optimization of matrix operations. Moreover, thanks to the modular design, this work can be easily extended to support other SCM-based channel models, hence providing a common framework for the simulation of wireless systems.

\section{ACKNOWLEDGMENTS}

The work of Tommaso Zugno was partially funded by the Google Summer of Code 2019 program. Also, the work of the authors from the University of Padova has been partially supported by NIST 
through Awards No. 70NANB17H166 and 70NANB18H273, and CTTC authors have received funding from the Spanish MINECO grant TEC2017-88373-R (5G-REFINE) and Generalitat de Catalunya grant 2017 SGR 1195. Finally, the authors would like to thank Tom Henderson for his useful suggestions and support during this work.

\section{REFERENCES}

[1] 3GPP. 2017. Study on Channel Model for Frequency Spectrum Above $6 \mathrm{GHz}$. TR 38.900 (Rel. 14). (2017)

[2] 3GPP. 2017. TR 38.913, Study on Scenarios and Requirements for Next Generation Access Technologies, V14.1.0. (2017)

[3] 3GPP. 2018. NR and NG-RAN Overall Description. TS 38.300 (Rel. 15). (2018).

[4] 3GPP. Jun. 2019. Study on Channel Model for Frequencies from 0.5 to $100 \mathrm{GHz}$ TR 38.901 (Rel. 15), V15.0.0. (Jun. 2019)

[5] J. G. Andrews, T. Bai, M. N. Kulkarni, A. Alkhateeb, A. K. Gupta, and R. W. Heath. 2017. Modeling and Analyzing Millimeter Wave Cellular Systems. IEEE Transactions on Communications 65, 1 (Jan 2017), pp. 403-430.

[6] H. Assasa and J. Widmer. 2017. Extending the IEEE 802.11 ad Model: Scheduled Access, Spatial Reuse, Clustering, and Relaying. In Proceedings of the Workshop on $n s-3$ (WNS3 '17). ACM, Porto, Portugal, pp. 39-46.

[7] H. Assasa, J. Widmer, T. Ropitault, A. Bodi, and N. Golmie. 2019. High Fidelity Simulation of IEEE 802.11ad in ns-3 Using a Quasi-Deterministic Channel Model In Proceedings of the 2019 Workshop on Next-Generation Wireless with ns-3 (WNGW 2019). ACM, Florence, Italy, pp. 22-25.

[8] H. Assasa, J. Widmer, T. Ropitault, and N. Golmie. 2019. Enhancing the ns-3 IEEE 802.11ad Model Fidelity: Beam Codebooks, Multi-Antenna Beamforming Training, and Quasi-Deterministic MmWave Channel. In Proceedings of the 2019 Workshop on $n s-3$ (WNS3 2019). ACM, Florence, Italy, pp. 33-40.

[9] N. Baldo and M. Miozzo. 2009. Spectrum-Aware Channel and PHY Layer Modeling for ns-3. In Proceedings of the Fourth International ICST Conference on Performance Evaluation Methodologies and Tools (VALUETOOLS 2009). ICST, Pisa, Italy.

[10] F. Boccardi, R. W. Heath Jr, A. Lozano, T. L. Marzetta, and P. Popovski. 2014. Five Disruptive Technology Directions for 5G. IEEE Communication Magazine 52, 2 (Feb. 2014), pp. 74-80.

[11] F. Boccardi, H. Shokri-Ghadikolaei, G. Fodor, E. Erkip, C. Fischione, M. Kountouris, P. Popovski, and M. Zorzi. 2016. Spectrum Pooling in MmWave Networks: Opportunities, Challenges, and Enablers. IEEE Communications Magazine 54, 11 (November 2016), pp. 33-39.

[12] B. Bojovic, L. Giupponi, Z. Ali, and M. Miozzo. 2019. Evaluating Unlicensed LTE Technologies: LAA vs LTE-U. IEEE Access 7 (2019), pp. 89714-89751.

[13] Q. Chen, X. Xu, and H. Jiang. 2019. Spatial Multiplexing Based NR-U and WiFi Coexistence in Unlicensed Spectrum. In 2019 IEEE 90th Vehicular Technology Conference (VTC2019-Fall). Honolulu, HI, USA, pp. 1-5.

[14] P. Ferrand, M. Amara, S. Valentin, and M. Guillaud. 2016. Trends and Challenges in Wireless Channel Modeling for Evolving Radio Access. IEEE Communication Magazine 54, 7 (July 2016), pp. 93-99.

[15] K. Z. Ghafoor, M. Guizani, L. Kong, H. S. Maghdid, and K. F. Jasim. 2019. Enabling Efficient Coexistence of DSRC and C-V2X in Vehicular Networks. IEEE Wireless Communications (Early Access) (2019), pp. 2-8.

[16] I. Hemadeh, K. Satyanarayana, M. El-Hajjar, and L. Hanzo. 2018. Millimeter-Wave Communications: Physical Channel Models, Design Considerations, Antenna Constructions and Link-Budget. IEEE Communications Surveys Tutorials 20, 2 (2018), pp. 870 - 913.

[17] E. Khorov, A. Kiryanov, A. Lyakhov, and G. Bianchi. 2019. A Tutorial on IEEE 802.11ax High Efficiency WLANs. IEEE Communications Surveys Tutorials 21, 1 (Firstquarter 2019), pp. 197-216.

[18] O. H. Koymen, A. Partyka, S. Subramanian, and J. Li. 2015. Indoor mm-Wave Channel Measurements: Comparative Study of $2.9 \mathrm{GHz}$ and $29 \mathrm{GHz}$. In IEEE Global Communications Conference (GLOBECOM). San Diego, CA, USA, pp. 1-6.

[19] S. Lagen, L. Giupponi, S. Goyal, N. Patriciello, B. Bojovic, A. Demir, and M. Beluri. 2020. New Radio Beam-based Access to Unlicensed Spectrum: Design Challenges and Solutions. IEEE Communications Surveys Tutorials 22, 1 (Mar. 2020), pp. 8-37.

[20] S. Lagen, K. Wanuga, H. Elkotby, S. Goyal, N. Patriciello, and L. Giupponi. 2020 New Radio Physical Layer Abstraction for System-Level Simulations of 5G Networks. In Proceedings of IEEE International Conference on Communications (IEEE ICC). Dublin, Ireland.

[21] L. Lanante, S. Roy, S. E. Carpenter, and S. Deronne. 2019. Improved Abstraction for Clear Channel Assessment in ns-3 802.11 WLAN Model. In Proceedings of the 2019 Workshop on ns-3 (WNS3 2019). Florence, Italy, pp. 49-56.
[22] M. Lecci, P. Testolina, M. Giordani, M. Polese, T. Ropitault, C. Gentile, N. Varshney, A. Bodi, and M. Zorzi. 2020. Simplified Ray Tracing for the Millimeter Wave Channel: A Performance Evaluation. In Information Theory and Applications Workshop (ITA). San Diego, CA, USA.

[23] R. Maldonado, C. Rosa, and K. I. Pedersen. 2020. Latency and Reliability Analysis of Cellular Networks in Unlicensed Spectrum. IEEE Access 8 (2020), pp. 4941249423.

[24] A. Maltsev, A. Pudeyev, A. Lomayev, and I. Bolotin. 2016. Channel Modeling in the Next Generation mmWave Wi-Fi: IEEE 802.11ay Standard. In 22th European Wireless Conference. Oulu, Finland.

[25] M. Mezzavilla, M. Zhang, M. Polese, R. Ford, S. Dutta, S. Rangan, and M. Zorzi. 2018. End-to-End Simulation of $5 \mathrm{G}$ mmWave Networks. IEEE Communications Surveys \& Tutorials 20, 3 (Third quarter 2018), pp. 2237-2263.

[26] N. Patriciello, S. Goyal, S. Lagen, L. Giupponi, B. Bojovic, A. Demir, M. Beluri. 2019. NR-U and WiGig Coexistence in $60 \mathrm{GHz}$ Bands. arXiv preprint arXiv:2001.04779. (2019).

[27] G. Naik, B. Choudhury, and J. Park. 2019. IEEE 802.11bd 5G NR V2X: Evolution of Radio Access Technologies for V2X Communications. IEEE Access 7 (2019), pp. 70169-70184.

[28] P. Nuggehalli. 2016. LTE-WLAN Aggregation [Industry Perspectives]. IEEE Wireless Communications 23, 4 (Aug. 2016), pp. 4-6.

[29] N. Patriciello, S. Lagen, B. Bojovic, and L. Giupponi. 2019. An E2E Simulator for 5G NR Networks. Simulation Modelling Practice and Theory 96 (2019), pp. 101933.

[30] M. Polese, F. Restuccia, A. Gosain, J. Jornet, S. Bhardwaj, V. Ariyarathna, S. Mandal, K. Zheng, A. Dhananjay, M. Mezzavilla, J. Buckwalter, M. Rodwell, X. Wang, M. Zorzi, A. Madanayake, and T. Melodia. 2019. MillimeTera: Toward A Large-Scale Open-Source MmWave and Terahertz Experimental Testbed. In Proceedings of the 3rd ACM Workshop on Millimeter-Wave Networks and Sensing Systems (mmNets '19). ACM, Los Cabos, Mexico, pp. 27-32.

[31] M. Polese and M. Zorzi. 2018. Impact of Channel Models on the End-to-End Performance of mmWave Cellular Networks. In IEEE 19th International Workshop on Signal Processing Advances in Wireless Communications (SPAWC). Kalamata, Greece.

[32] T. S. Rappaport, S. Sun, R. Mayzus, H. Zhao, Y. Azar, K. Wang, G. N. Wong, J. K. Schulz, M. Samimi, and F. Gutierrez. 2013. Millimeter Wave Mobile Communications for 5G Cellular: It Will Work! IEEE Access 1 (May 2013), pp. 335-349.

[33] M. Rebato, F. Boccardi, M. Mezzavilla, S. Rangan, and M. Zorzi. 2017. Hybrid Spectrum Sharing in mmWave Cellular Networks. IEEE Transactions on Cognitive Communications and Networking 3, 2 (June 2017), pp. 155-168.

[34] M. Rebato, M. Polese, and M. Zorzi. 2018. Multi-Sector and Multi-Panel Performance in 5G mmWave Cellular Networks. In 2018 IEEE Global Communications Conference (GLOBECOM). Abu Dhabi, United Arab Emirates, pp. 1-6.

[35] K. A. Remley, J. A. Gordon, D. Novotny, A. E. Curtin, C. L. Holloway, M. T. Simons, R. D. Horansky, M. S. Allman, D. Senic, M. Becker, J. A. Jargon, P. D. Hale, D. F. Williams, A. Feldman, J. Cheron, R. Chamberlin, C. Gentile, J. Senic, R. Sun, P. B. Papazian, J. Quimby, M. Mujumdar, and N. Golmie. 2017. Measurement Challenges for $5 \mathrm{G}$ and Beyond: An Update from the National Institute of Standards and Technology. IEEE Microwave Magazine 18, 5 (July 2017), pp. 41-56.

[36] S. K. Saha, Y. Ghasempour, M. K. Haider, T. Siddiqui, P. De Melo, N. Somanchi, L. Zakrajsek, A. Singh, R. Shyamsunder, O. Torres, et al. 2019. X60: A Programmable Testbed for Wideband $60 \mathrm{GHz}$ WLANs with Phased Arrays. Computer Communications 133 (Jan. 2019), pp. 77-88.

[37] A. A. M. Saleh and R. Valenzuela. 1987. A Statistical Model for Indoor Multipath Propagation. IEEE fournal on Selected Areas in Communications 5, 2 (February 1987), pp. $128-137$.

[38] Q. H. Spencer, C. B. Peel, A. L. Swindlehurst, and M. Haardt. 2004. An Introduction to the Multi-User MIMO Downlink. IEEE Communications Magazine 42, 10 (Oct 2004), pp. 60-67.

[39] P. Testolina, M. Lecci, M. Polese, M. Giordani, and M. Zorzi. 2020. Scalable and Accurate Modeling of the Millimeter Wave Channel. In International Conference on Computing, Networking and Communications (ICNC).

[40] WBA and NGMN Alliance. 2019. RAN Convergence Paper. version 1.0. (Aug. 2019).

[41] M. Zhang, M. Polese, M. Mezzavilla, S. Rangan, and M. Zorzi. 2017. ns-3 Implementation of the 3GPP MIMO Channel Model for Frequency Spectrum above 6 GHz. In Proceedings of the Workshop on ns-3 (WNS3 2017). ACM, Porto, Portugal, pp. 71-78.

[42] P. Zhou, K. Cheng, X. Han, X. Fang, Y. Fang, R. He, Y. Long, and Y. Liu. 2018. IEEE 802.11ay-Based mmWave WLANs: Design Challenges and Solutions. IEEE Communications Surveys Tutorials 20, 3 (thirdquarter 2018), pp. 1654-1681. 